Accepted to the Astrophysical Journal on October 3, 2013

Preprint typeset using LTEX style emulateapj v. 5/2/11

\title{
THE XMM-NEWTON SPECTRUM OF A CANDIDATE RECOILING SUPERMASSIVE BLACK HOLE: AN ELUSIVE INVERTED P-CYGNI PROFILE
}

\author{
G. Lanzuisi ${ }^{1,2}$, F. Civano ${ }^{1,3}$, S. Marchesi ${ }^{1,4}$, A. Comastri ${ }^{5}$, E. Costantini ${ }^{6}$, M. Elvis $^{3}$, V. Mainieri ${ }^{7}$, R. Hickox ${ }^{1}$, K. JAhnke $^{8}$, S. Komossa $^{9}$, E.

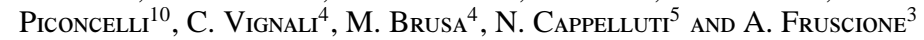 \\ Accepted to the Astrophysical Journal on October 3, 2013
}

\begin{abstract}
We present a detailed spectral analysis of new XMM-Newton data of the source CXOC J100043.1+020637, also known as CID-42, detected in the COSMOS survey at $\mathrm{z}=0.359$. Previous works suggested that CID42 is a candidate recoiling supermassive black holes showing also an inverted P-Cygni profile in the X-ray spectra at $\sim 6 \mathrm{keV}$ (rest) with an iron emission line plus a redshifted absorption line (detected at $3 \sigma$ in previous XMM-Newton and Chandra observations). Detailed analysis of the absorption line suggested the presence of ionized material inflowing into the black hole at high velocity. In the new long XMM-Newton observation, while the overall spectral shape remains constant, the continuum 2-10 keV flux decreased of $\sim 20 \%$ with respect to previous observation and the absorption line is undetected. The upper limit on the intensity of the absorption line is $\mathrm{EW}<162 \mathrm{keV}$. Extensive Monte Carlo simulations show that the non detection of the line is solely due to variation in the properties of the inflowing material, in agreement with the transient nature of these features, and that the intensity of the line is lower than the previously measured with a probability of $98.8 \%$. In the scenario of CID-42 as recoiling SMBH, the absorption line can be interpreted as due to inflow of gas with variable density and located in the proximity of the SMBH and recoiling with it. New monitoring observations will be requested to further characterize this line.
\end{abstract}

Subject headings: galaxies: active - surveys $-\mathrm{X}$-rays:galaxies

\section{INTRODUCTION}

During galaxy major mergers, the central supermassive black holes (SMBHs) that reside within the merging galaxies will form a bound binary that can itself merge (e.g. Volonteri et al. 2003, Hopkins et al. 2008, Colpi \& Dotti 2009). At the time of the SMBH binary coalescence, strong gravitational wave (GW) radiation is emitted an-isotropically, depending on the spin and mass-ratio of the two SMBHs. To conserve linear momentum, the newly formed single SMBH recoils (Peres 1962, Bekenstein 1973). Recoiling SMBHs are the direct products of processes in the strong field regime of gravity and are one of the key observable signatures of a SMBH binary merger. As the SMBH recoils from the center of the galaxy, the closest regions (disk and broad line regions) are carried with it and the more distant region are left behind depending on the recoil velocity (Merritt et al. 2006, Loeb 2007). Because GW recoil displaces (or ejects) SMBHs from

\footnotetext{
${ }^{1}$ Department of Physics and Astronomy, Dartmouth College, Wilder Laboratory, 03855 Hanover, NH, USA

${ }^{2}$ Institute of Astronomy \& Astrophysics, National Observatory of Athens, Palaia Penteli, 15236, Athens, Greece

${ }^{3}$ Smithsonian Astrophysical Observatory, 60 Garden st., 02138 Cambridge, MA, USA

${ }^{4}$ Dipartimento di Astronomia, Universitá degli Studi di Bologna, via Ranzani 1, 40127, Bologna, Italy

5 INAF- Osservatorio Astronomico di Bologna, via Ranzani 1, 40127. Bologna, Italy

${ }^{6}$ SRON, Netherlands Institute for Space Research, Sorbonnelaan, 2, 3584 CA, Utrecht, The Netherlands

${ }^{7}$ European Southern Observatory, Karl-Schwarschild-Strasse 2, 85748, Garching bei Munchen, Germany

${ }^{8}$ Max Planck Institute for Astronomy, Konigstuhl 17, D-69117 Heidelberg, Germany

${ }^{9}$ Max-Planck-Institut fuer Radioastronomie, Auf dem Huegel 69, 53121 Bonn, Germany

${ }^{10}$ INAF-Osservatorio Astronomico di Roma, via Frascati 33, 00040 Monteporzio Catone, Italy
}

the centers of galaxies, these events have the potential to influence the observed co-evolution of SMBHs with their host galaxies, as demonstrated by numerical simulations (Blecha et al. 2011, Sijacki et al. 2011, Guedes et al. 2011). Observational searches for recoiling SMBHs are just at the beginning (Bonning et al. 2007, Eracleous et al. 2012, see Komossa 2012 for a review) and only few serendipitous discoveries of candidates have been reported in the literature (Komossa et al. 2008, Shields et al. 2009, Robinson et al. 2010, Jonker et al. 2010, Batcheldor et al. 2010, Steinhardt et al. 2012) .

The Chandra-COSMOS source CXOC J100043.1+020637 $(z=0.359$, Elvis et al. 2009, Civano et al. 2012), also known as CID-42, is a candidate for being a GW recoiling SMBH with both imaging (in optical and X-ray) and spectroscopic signatures (Civano et al. 2010, 2012; hereafter C10 and C12). The current data are consistent with a recoiling SMBH ejected 1-6 Myr ago, as shown by detailed modeling presented in Blecha et al. (2013).

In both the Chandra and XMM-Newton spectra of CID-42, a remarkable inverted $P$-Cygni profile, i.e., an absorption feature redshifted with respect to the emission component, was detected by $\mathrm{C} 10$ at $\sim 4.5 \mathrm{keV}$ in the observed frame and $\sim 6$ $\mathrm{keV}$ in the rest frame (Fig. 1 and 2 adapted from Fig. 7 of $\mathrm{C} 10)$. The emission feature was consistent with being a neutral iron line at the system redshift, with a constant flux but more prominent in the Chandra spectrum $(\mathrm{EW}=570 \pm 260$ $\mathrm{eV})$ than in the XMM-Newton one $\left(\mathrm{EW}=142_{-86}^{+143} \mathrm{eV}\right)$. The absorption feature (at $\sim 6 \mathrm{keV}$ in the rest frame), detected in both XMM-Newton-EPIC pn and Chandra-ACIS spectra, showed a line energy centroid changing in time between 5.8 and 6.2 $\mathrm{keV}\left(\Delta \mathrm{E}_{\text {rest }} \sim 500 \mathrm{eV}\right.$; see Figure 8 of $\left.\mathrm{C} 10\right)$, with intensity of $350 \pm 120 \mathrm{eV}$. A re-analysis of C10 data, including extensive Monte Carlo simulations (see Section 4.1), shows that the significance of the redshifted absorption line in the XMMNewton data is $3 \sigma$, strengthened by the detection of the line 
also in Chandra-ACIS at 2.2 $\sigma$ (Figure2).

Most known X-ray absorbers in Active Galactic Nuclei (AGN) are observed as blueshifted lines and so are signatures of fast outflowing winds (see Tombesi et al. 2013 and references therein) predicted in both phenomenological and semianalytical quasar models (e.g., Elvis 2000, King \& Pounds 2003, King 2010). Redshifted absorbers, instead, would seem to require high-velocity inflows, which must therefore be located in the proximity of the SMBH.

Few cases of objects with redshifted absorption lines are reported in the literature (NGC 3516, Nandra et al. 1999; E1821+643, Yaqoob \& Serlemitsos 2005; Mrk 509, Dadina et al. 2005; PG 1211+143, Reeves et al. 2005; Q0056-363, Matt et al. 2005; Ark 120, Nandra et al. 2007; Mrk 335, Longinotti et al. 2007). In most cases, the redshifted absorption line has not been observed in additional observations (Vaughan \& Uttley 2008 for an extended discussion on the statistical significance of these lines). As for example, the redshifted absorption line in Mrk 335 was reported by Longinotti et al. (2007). Later paper with new data for this source do not search for the redshifted absorption line previously detected (O' Neill et al. 2007, Grupe et al. 2012). The redshifted line seen in Mrk 509 with Beppo-SAX (Dadina et al. 2005) was not detected again by Cappi et al. (2009) in 5 XMMNewton observations taken between 2000 and 2006. Ponti et al. (2013) observed again Mrk 509 in 2009 as part of a long XMM-Newton campaign $(10 \times 60 \mathrm{ks}$ observations $)$ but the line was reported as undetected again. In PG 1211+143, a redshifted absorption line was first observed (Reeves et al. 2005) however in newer data a classic P-Cygni profile only is reported (Pounds et al. 2009). Q0056-363 was observed in 2000 and in 2003: both the observations are discussed in Matt et al. (2005). The redshifted absorption line was detected only in 2003 data. Moreover, this observation has been split in five time-intervals in order to study short time-scale variability: the absorption line was found in only one of these intervals. Tombesi et al. (2010) also reported the detection (at a 90\% confidence level using the F-test) of redshifted absorption lines in seven more sources (including Mrk 335). All these evidences suggest that these features are highly variable, and that their occurrence, or duty cycle, is very low.

The most convincing case is E1821+643, a candidate recoiling SMBH (Robinson et al., 2010), like CID-42, with also an iron redshifted absorption line in its Chandra X-ray spectrum, observed by Yaqoob \& Serlemitsos (2005). E1821+643 was observed again with XMM-Newton by Jiménez-Bailón et al. (2007), who reported a new measure for the peak energy of the absorption line. The absorption line has been explained as gravitationally redshifted iron absorption and its line energy variability as changes in the ionization state of the iron clouds.

C10 suggested a possible interpretation for the redshifted absorption line seen in CID-42 as gas (either neutral or ionized iron) infalling into the recoiling $\mathrm{SMBH}$ at relativistic velocities $(0.02-0.14 c)$, but, given the data quality and the degeneracy between velocity and ionization state, a firm conclusion on the properties of the infalling material could not be reached.

In order to perform an accurate characterization of the Xray absorber (density, velocity, covering factor and ionization state), we requested and obtained a $123 \mathrm{ks}$ long un-interrupted XMM-Newton observations. In this paper, we present the analysis of the line profile as well as the broad band spectral shape and the obtained results.
Throughout the paper $90 \%$ errors $(1.6 \sigma)$ are quoted unless otherwise stated.

\section{DATA REDUCTION}

CID-42 was observed by XMM-Newton with a $123 \mathrm{ks}$ single observation taken on June 3, 2011 (during revolution 2103, observation ID 0672780101, PI: F. Civano). Standard XMM-Newton SAS tasks epproc and emproc (SAS 12.0.1) were used to produce calibrated pn and MOS event file. These event files were processed using the latest calibration database and cleaned up from hot pixels and cosmic rays contamination. X-ray events corresponding to patterns 0-4 (0-12) for the pn (MOS) cameras were selected.

A global light-curve at energies greater than $10 \mathrm{keV}$ (where the contribution from the emission of X-ray sources is negligible) was produced in order to identify any background flare (Figure 3). The last $\sim 15 \mathrm{ks}$ of the observation are affected by high background ( $25 \mathrm{ct} / \mathrm{s})$, typical of the edge of each XMM-Newton observing orbit. Moreover, the central part of the observation has a long $(\sim 50 \mathrm{ks})$ interval during which the background intensity is 10 times stronger than the remaining time in this observation.

For the purposes of this work (i.e., study the absorption feature), two different approaches have been used in filtering the data to remove the flare: (A) to maximize the number of counts, (B) to maximize the signal-to-noise ratio. In the less conservative case (A), a standard $3 \sigma$ clipping method has been used to discard intervals with high background rate (dotted line in Fig. 3). The part of the observation where the background contribution is lower than $5 \mathrm{cts} \mathrm{s}^{-1}\left(0.5 \mathrm{cts} \mathrm{s}^{-1}\right)$ in the pn (MOS) has been included. In the more conservative case (B), only the time intervals with background intensity $<0.5(<0.15)$ cts $^{-1}$ in the pn (MOS) have been considered for the analysis (dashed line in Fig. 3), obtained by applying twice a $3 \sigma$ clipping cut. This level is consistent with the one used in Cappelluti et al. (2007) to clean the XMM-Newton data used in $\mathrm{C} 10$ analysis. After the cleaning operation, a net exposure time of $\sim 100(\sim 110) \mathrm{ks}$ and $\sim 53(\sim 80) \mathrm{ks}$ is available for the pn (MOS) camera in case A and $\mathrm{B}$, respectively (see Table1).

Source and background spectra were extracted for both case A and B from the data of each camera. The source spectra were extracted from circular regions of radius $25^{\prime \prime}$ (MOS1 and MOS2) and 30" (pn), maximizing the number of source counts and minimizing the background contribution. The background spectra were extracted from multiple regions close to the target, avoiding CCD gaps and other X-ray detected sources listed in the COSMOS X-ray catalogs (Cappelluti et al. 2009, Brusa et al. 2010, Elvis et al. 2009, Civano et al. 2012). The background extraction areas are typically about 20 times the source extraction region, in order to average out all the position-dependent background features and to obtain enough background counts and perform accurate spectral analysis. The background spectra are shown in Figure 4 as green and blue points.

Table 1 lists net counts in each camera in 3 bands (0.5-7, 0.5-2 and 2-7 keV), together with the same numbers for the pn spectrum reported in $\mathrm{C} 10$. In the same table we also report the fraction of source counts in the extraction region in the 0.5-7 $\mathrm{keV}$ band (column 5) and also in the $2-7 \mathrm{keV}$ band (column 6). The source signal-to-noise ratio in the $2-7 \mathrm{keV}$ band, where the absorption and emission lines were previously observed, is lower in case A spectra than in B, where the high background flare was removed. This effect is very important in the pn with 


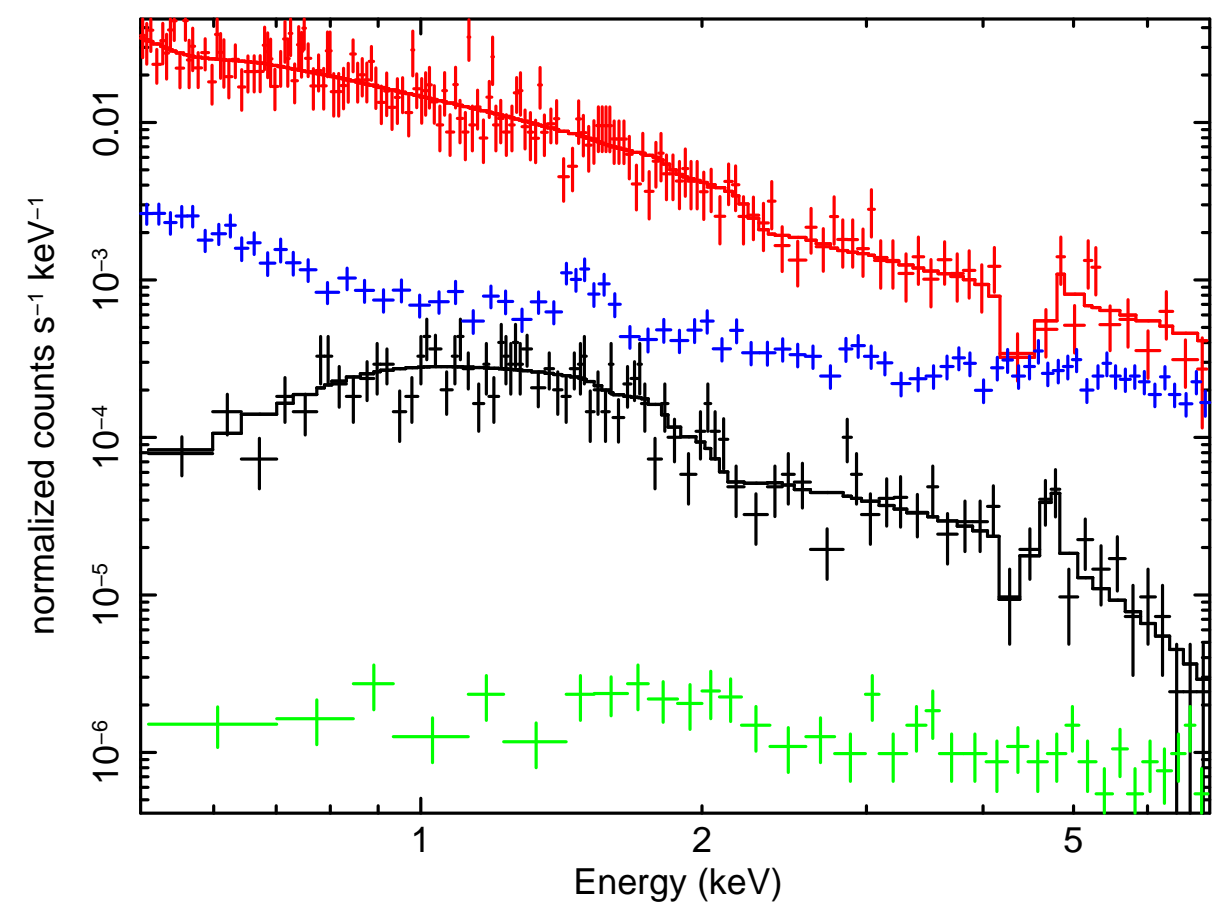

Figure 1. EPIC pn (red) and ACIS (black) total spectra of CID-42 adapted from Figure 7 of C10 and rebinned for plotting purposes. The background spectra (pn in blue and ACIS in green) are reported and rescaled to the source area. The y scale is arbitrary but the ratio between source and background has been preserved. The best fit model (absorbed power-law, thermal component and emission and absorption features) is plotted.

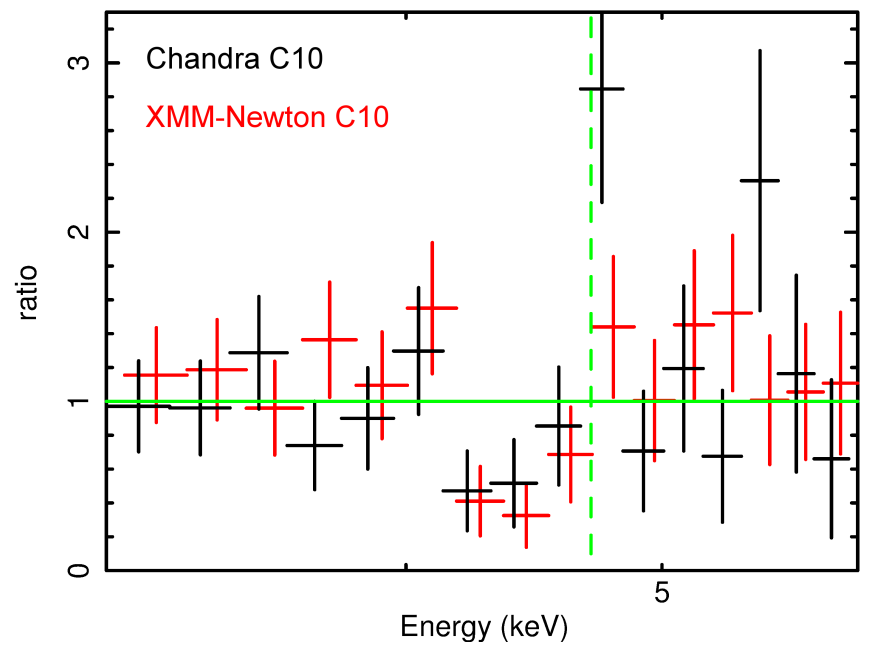

Figure 2. Data to model ratio around the energy where the inverted P-Cygni profile is detected in EPIC pn (red) and ACIS (black) spectra of CID- 42 adapted from C10. The model used here does not includes the emission and absorption features, only the continuum model is used. The vertical line corresponds to the $6.4 \mathrm{keV}$ rest frame energy. The spectra have been rebinned in energy with $\Delta \mathrm{E} \sim 200 \mathrm{eV}$ in order to highlight the absorption features.

a difference of $\sim 30 \%$ between $\mathrm{A}$ and $\mathrm{B}$ in the $2-7 \mathrm{keV}$ band. The net number of counts however is higher in case $A$ than in case B. In the next section, a discussion on the spectra choice is presented.

\section{SPECTRAL ANALYSIS}

To perform the spectral analysis, we followed the approach of $\mathrm{C} 10$, where the spectral fit is performed on the total spectrum, modeling the source and the background simultaneously (see Fiore et al. 2012 and Lanzuisi et al. 2013 for details on this approach), after first determining an accurate model for the background.
The XMM-Newton EPIC pn and MOS background spectra $(\sim 8000$ and $\sim 4000$ counts each in case A and B) were fitted over the $0.3-7 \mathrm{keV}$ range using XSPEC version 12.8 (Arnaud 1999) using the following components: two powerlaw components, a thermal component for the soft part of the spectrum, 3 Gaussian emission lines to reproduce the features of the pn and MOS background $\$$. This background best-fit model was then rescaled to the source area using the ratio of the extraction region areas (BACKSCAL keyword).

The source spectra were fitted in the $0.5-7 \mathrm{keV}$ band with

11 See the list of emission lines for both the pn and MOS camera at this web site http://wWw.star.le.ac.uk/ amr30/BG/BGTable.html 


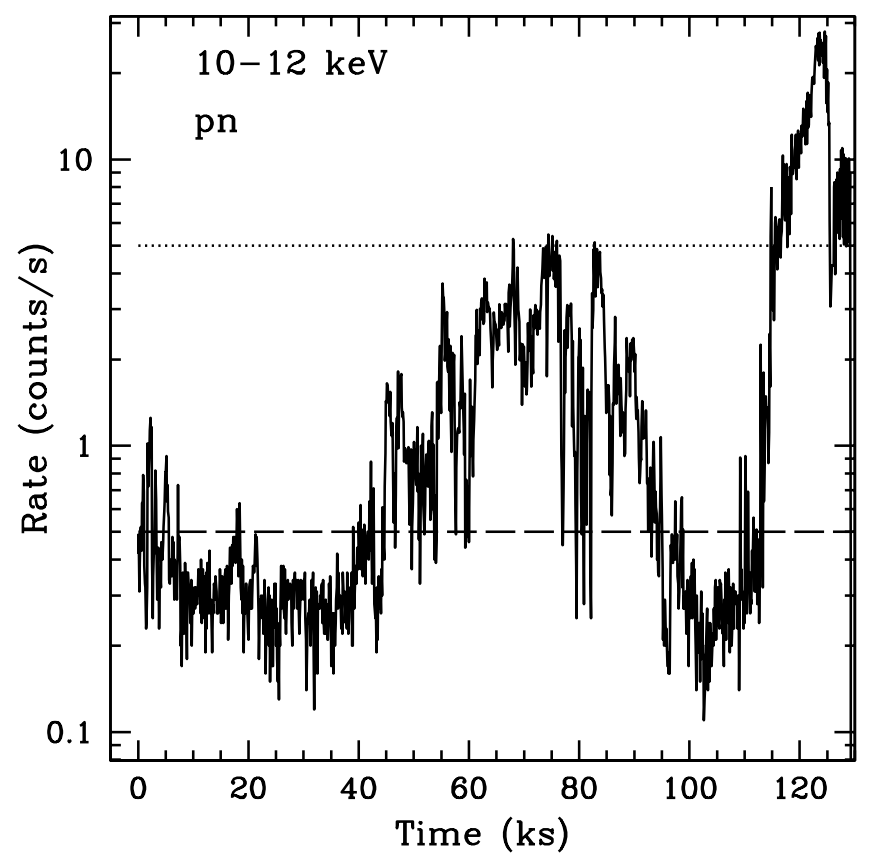

Figure 3. The 10-12 keV light curve extracted from the pn data. The dotted and dashed lines represents the cuts applied to the data in case A and B respectively.

Table 1

Exposure times and net counts in the three cameras after the cleaning operations. For a comparison, we also report the same values for the observation used in C10.

\begin{tabular}{cccccccc}
\hline \hline & Instrument & $\begin{array}{c}\text { Exposure time } \\
\mathrm{ks}\end{array}$ & $\begin{array}{c}\text { Counts } \\
0.5-7 \mathrm{keV}\end{array}$ & $\begin{array}{c}\text { Counts } \\
0.5-2 \mathrm{keV}\end{array}$ & $\begin{array}{c}\text { Counts } \\
2-7 \mathrm{keV}\end{array}$ & $\begin{array}{c}\text { Source \% } \\
0.5-7 \mathrm{keV}\end{array}$ & $\begin{array}{c}\text { Source \% } \\
2-7 \mathrm{keV}\end{array}$ \\
\hline \multirow{2}{*}{$\mathrm{A}$} & MOS1+MOS2 & 225.3 & 1950 & 1539 & 404 & 84.4 & 66.8 \\
& $\mathrm{pn}$ & 100.8 & 3013 & 2502 & 511 & 77.9 & 49.0 \\
\hline \multirow{2}{*}{$\mathrm{B}$} & MOS1+MOS2 & 160.3 & 1420 & 1106 & 314 & 86.8 & 75.4 \\
& $\mathrm{pn}$ & 52.77 & 1622 & 1370 & 251 & 87.2 & 68.1 \\
\hline $\mathrm{C} 10$ & $\mathrm{pn}$ & 71.2 & 1500 & 1237 & 280 & 87.1 & 76.4 \\
\hline \hline
\end{tabular}

an absorbed power-law plus a thermal component (mekal), the same model used in $\mathrm{C} 10$. A Galactic column density along the line of sight of $\mathrm{N}_{H, G a l}=2.6 \times 10^{20} \mathrm{~cm}^{-2}$ (Kalberla et al. 2005) has been included as well. The modified Cash statistic implemented in XSPEC (cstat; Cash 1979) has been employed for the fitting, and a minimum binning of 1 count per bin has been applied to the spectra. The pn and MOS1+MOS2 spectra were first fitted separately to verify the consistency of the fit between instruments, then jointly to more tightly constrain the errors on the spectral parameters. In Figure 4 the case A spectra are shown (top: pn with background spectrum; bottom: MOS with background spectrum). The best fits of the spectra in case A and B are fully consistent within the errors, however, in case A, where the number of source counts is higher, the parameters are better constrained.

The spectral analysis results for the joint fit are listed in Table 2 for case A together with the best fit parameters reported in $\mathrm{C} 10$. The spectral index obtained from the joint fit is $\Gamma=2.16 \pm 0.08$, slightly steeper than the slope measured in $\mathrm{C} 10$. The presence of a thermal component is significant at $>5 \sigma$, as indicated by the F-test, and the temperature is $k T=$ $0.18_{-0.04}^{+0.02} \mathrm{keV}$. An upper limit of $\mathrm{N}_{H}<6 \times 10^{20} \mathrm{~cm}^{-2}$ is measured for the intrinsic absorption.

The model fluxes, computed in the $0.5-2,2-10$ and $0.5-10$
$\mathrm{keV}$ bands, are reported in Table 2. The 0.5-10 keV band flux is compared in Figure 5 with the fluxes obtained in the previous XMM-Newton and Chandra observations (C10 and C12). The light curve spans a period of $\sim 9$ years. The flux reported here is consistent with the flux reported in $\mathrm{C} 10$ for the XMM-Newton data and consistent within the error with the C12 flux for the Chandra HRC observation taken in January 26,2011 . While the soft component of the spectrum remains almost constant, the hard part of the spectrum is $20 \%$ fainter here than in C10. The flux light curve of CID-42 has been analyzed in a variability study of the XMM-COSMOS sample by Lanzuisi et al. (submitted), where it is shown that CID-42 is among the $20 \%$ most variable sources among the brightest ( $>1000$ counts) 65 sources in their sample $(\sim 900$ sources total). The light curve of the source within the 2011 XMM-Newton observation studied here does not show short time scale variability.

\section{P-CYGNI PROFILE ANALYSIS}

The main goal of this work is to study the properties of the absorption feature that was detected in the previous XMMNewton and Chandra observations (see Section 4.1), however no clear evidence of it has been found in the new data. The iron emission line instead is still detected and prominent. In 


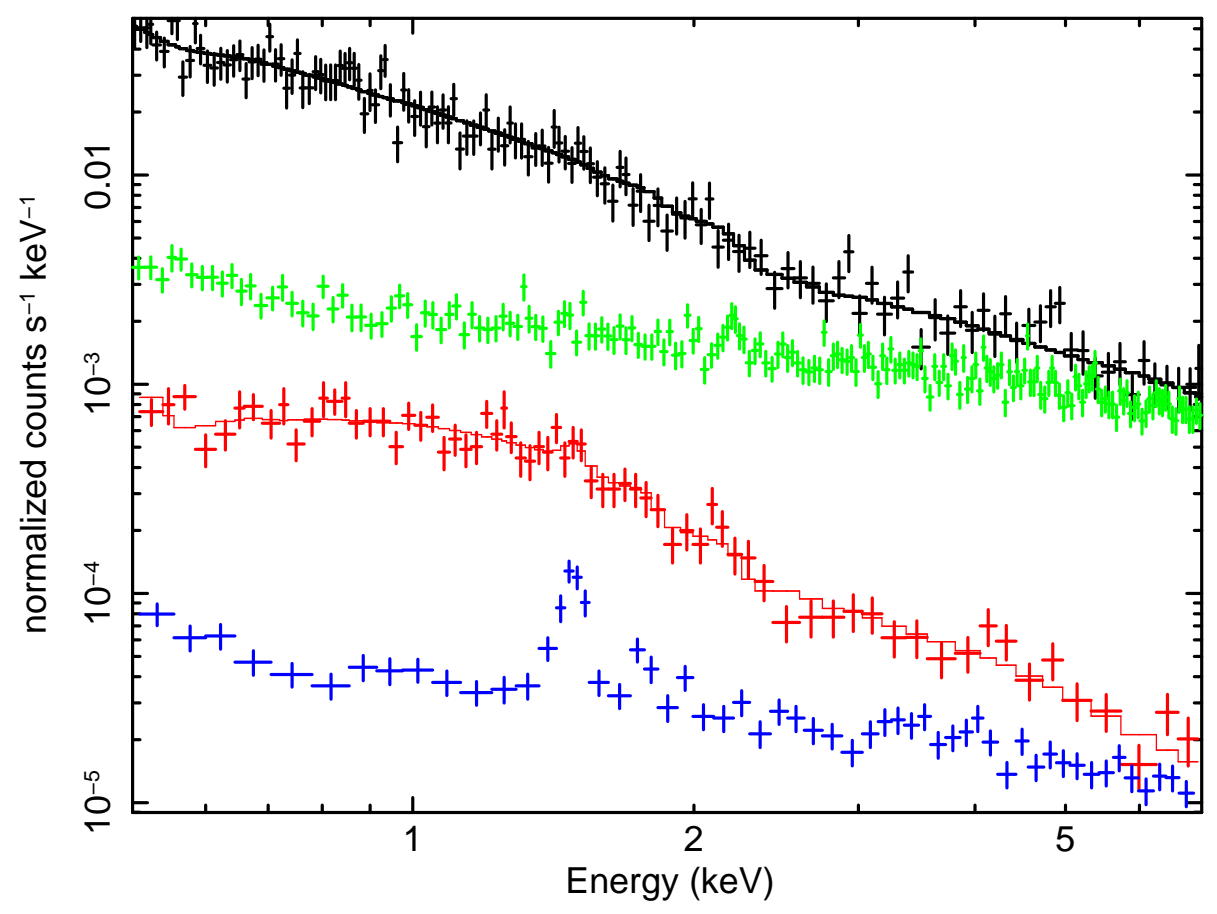

Figure 4. EPIC pn (black) and MOS1+MOS2 (red) total spectra (case A), rebinned for plotting purposes. The background spectra (pn in green and MOS in blue) are reported and rescaled to the source area. The y-axis normalization is arbitrary but the source to background ratio is preserved. The model is the best fit reported in Table 2 (no emission line is included).

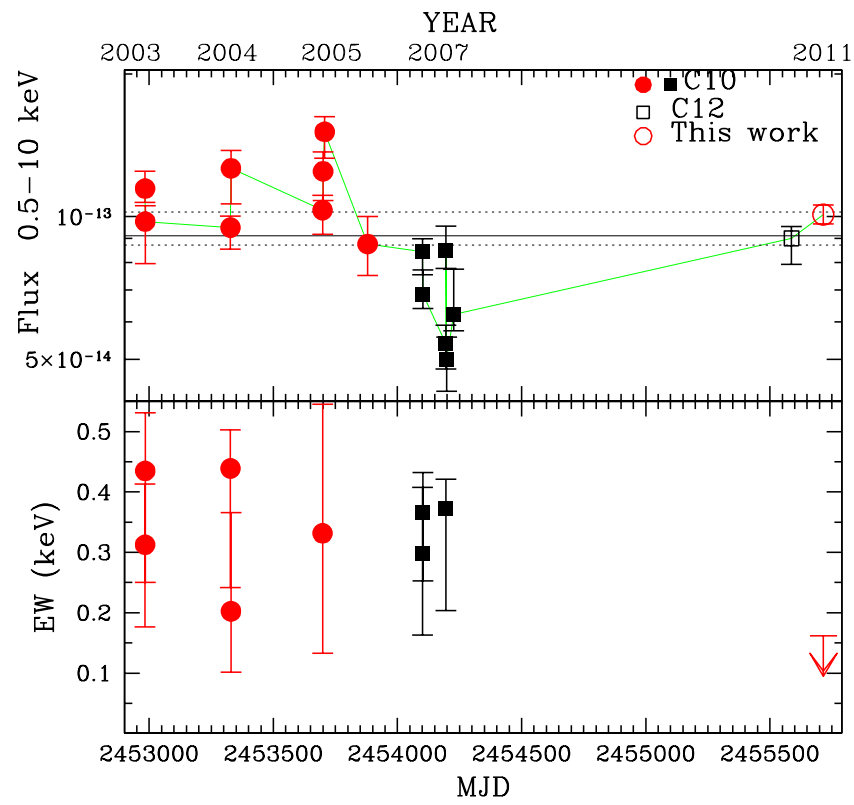

Figure 5. Top: X-ray full band flux light curve (red=XMM-Newton black=Chandra) adapted from C10, including the data point from the 2011 XMM-Newton data and also C12 Chandra HRC data. The reported errors are at 90\% confidence level. The mean value and error are reported as horizontal lines. Bottom: Absorption line equivalent width measured in different epochs spectra. 
Table 2

Spectral analysis results (continuum and emission line) and fluxes for the joint spectral fitting of pn and MOS1+MOS2 and the values from the ACIS-I and pn spectra analyzed in C10. Errors are at $90 \%$ confidence level.

\begin{tabular}{cccc}
\hline \hline Fit Parameter & ACIS-I (C10) & pn (C10) & pn+MOS (here) \\
\hline Continuum & & & \\
$\Gamma$ & $1.88_{-0.13}^{+0.17}$ & $1.95_{-0.06}^{+0.07}$ & $2.16_{-0.08}^{+0.08}$ \\
$N_{H}\left(10^{22} \mathrm{~cm}^{-2}\right)$ & $<0.2$ & $<0.02$ & $<0.06$ \\
$k T(\mathrm{keV})$ & $<0.11$ & $<0.13$ & $0.18_{-0.04}^{+0.02}$ \\
$\mathrm{~F}_{0.5-2 \mathrm{keV}}\left(10^{-14} \mathrm{erg} \mathrm{cm}^{-2} \mathrm{~s}^{-1}\right)$ & $1.8 \pm 0.2$ & $4.8 \pm 0.3$ & $5.1 \pm 0.2$ \\
$\mathrm{~F}_{2-10 \mathrm{kV}}\left(10^{-14} \mathrm{erg} \mathrm{cm}^{-2} \mathrm{~s}^{-1}\right)$ & $2.9 \pm 0.3$ & $6.1 \pm 0.4$ & $4.9 \pm 0.3$ \\
$\mathrm{~F}_{0.5-10 \mathrm{keV}}\left(10^{-14} \mathrm{erg} \mathrm{cm}^{-2} \mathrm{~s}^{-1}\right)$ & $5.1 \pm 0.4$ & $10.9 \pm 0.6$ & $10.1 \pm 0.5$ \\
\hline Emission Line (one Gaussian fit) & & & \\
Observed Energy & $6.44 \pm 0.07$ & $6.60 \pm 0.15$ & $6.62_{-0.09}^{+0.12}$ \\
Line $\sigma$ (keV) & $<0.12$ & $<0.2$ & $<0.6$ \\
EW (eV) & $570 \pm 260$ & $142_{-86}^{+143} \mathrm{eV}$ & $593_{-390}^{+347}$ \\
\hline Emission Line (double Gaussian fit) & & & \\
Observed Energy & $\ldots \ldots \ldots \ldots$ & $\ldots \ldots \ldots$ & $6.70_{-0.13}^{+0.11}$ \\
Line $\sigma(\mathrm{keV})$ & $\ldots \ldots \ldots \ldots$ & $\ldots \ldots \ldots$ & $<0.2$ \\
EW (eV) & $\ldots \ldots \ldots \ldots$ & $\ldots \ldots \ldots$ & $394_{-160}^{+277}$ \\
Observed Energy & $\ldots \ldots \ldots \ldots$ & $\ldots \ldots \ldots$ & $6.41_{-0.30}^{+0.37}$ \\
Line $\sigma(\mathrm{keV})$ & $\ldots \ldots \ldots \ldots$ & $\ldots \ldots \ldots$ & $<0.2$ \\
EW (eV) & $\ldots \ldots \ldots \ldots$ & $\ldots \ldots \ldots$ & $<500$ \\
\hline \hline
\end{tabular}

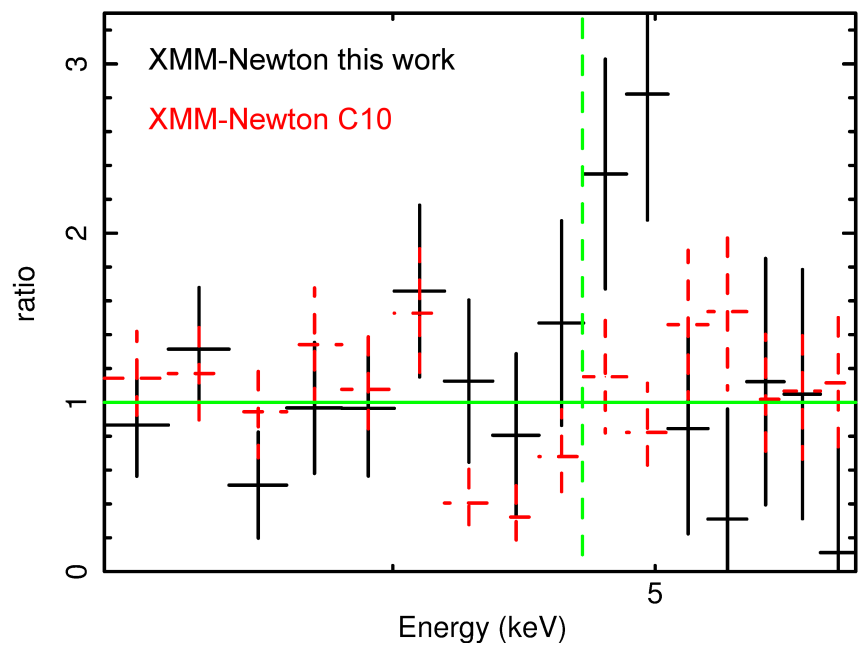

Figure 6. A zoom on the data to model (no features included, only the continuum model is used) ratio around the energy of the inverted $P$-Cygni profile. C10 pn data (red dashed line) are compared to the new pn data (black solid). The vertical line corresponds to the $6.4 \mathrm{keV}$ rest frame energy. The data in the zoom have been rebinned in energy with $\Delta \mathrm{E}=200 \mathrm{eV}$ to highlight the presence of the absorption feature. 
Figure 6, the ratio between data and model of the new pn data with those presented in $\mathrm{C} 10$ is shown (the expected energy of the $6.4 \mathrm{keV} \mathrm{Fe} \mathrm{K} \alpha$ line is plotted).

Both the A and B spectra were used for the search of this line and both analyses do not return a detection. The analysis of spectrum B returns only a limit of $<248 \mathrm{eV}$ (90\% confidence level) on the equivalent width of the absorption line. The spectrum A, having $60 \%$ more counts than spectrum B in the hard band, returns a tighter limit on the equivalent width of $\mathrm{EW}<162 \mathrm{eV}$. The above upper limits have been computed at the energy where the absorption line was detected in C10 (4.5 $\mathrm{keV}$ observed frame). Given that, as discussed in the introduction, the line centroid has been observed to change between 4.2 and $4.6 \mathrm{keV}$ in the observed frame, it might be slightly different from the nominal value reported in C10. The upper limits fixing the line centroid in the $4-4.7 \mathrm{keV}$ observed range are consistent within $\pm 20 \mathrm{eV}$ (\%10) with the above values. This finding is consistent with the observed flat spectral slope measured on the broad band spectrum. In Figure 5 (bottom panel), the EW value measured is plotted as un upper limit together with the intensities measured in C10 XMM-Newton and Chandra spectra.

The emission line was first fitted with a narrow unresolved Gaussian line in both A and B (the numbers listed afterward are for spectrum $A$ and are reported in 2). The line centroid is higher than what expected for the neutral $\mathrm{FeK} \alpha$ line at 6.4 $\mathrm{keV}$ : the best fit rest frame energy in the joint fit is $6.62_{-0.09}^{+0.12}$ $\mathrm{keV}$, consistent with the value reported in $\mathrm{C} 10$ of $6.60_{-0.12}^{+0.15}$ $\mathrm{keV}$ for the XMM-Newton spectrum. In the Chandra spectrum instead, C10 reported an energy of $6.44 \pm 0.07 \mathrm{keV}$. The equivalent width of the line is $593_{-390}^{+347} \mathrm{eV}$ (pn value), brighter than the XMM-Newton value reported in C10 of $142_{-86}^{+143} \mathrm{eV}$ and comparable to the Chandra value $\left(E W_{\text {Chandra }}=570 \pm 260\right.$ $\mathrm{eV}$ ).

Leaving the emission line width free to vary, the profile width is $\sigma<0.6 \mathrm{keV}$ and can be fitted by using two narrow Gaussian lines, with rest frame energies of $6.70_{-0.13}^{+0.11}$ and $6.41_{-0.30}^{+0.37} \mathrm{keV}$ and equivalent width of $394_{-160}^{+278} \mathrm{eV}$ and $<500$ $\mathrm{eV}$ respectively (obtained by fixing the energy at the best fit). The emission line can be interpreted as the blended emission of the 6.4 and $6.7 \mathrm{keV}$ iron emission lines (neutral and He-like iron), however the two fits (one or two lines) are statistically indistinguishable.

In $\mathrm{C} 10$, the iron emission line is consistent with a single narrow line in both Chandra and XMM-Newton spectra. No hint of a second emission line or of a broad profile is observed. While the emission line in the Chandra spectrum is perfectly consistent with a neutral $\mathrm{Fe} \mathrm{K} \alpha$ line at $6.4 \mathrm{keV}$, the energy observed in the XMM-Newton spectrum is higher $(6.6 \mathrm{keV})$. This can be however interpreted as the effect of the broader absorption feature in XMM-Newton that allows only the blue wing of the line to be observed (Fig. 1 and 2).

\subsection{Statistical significance of absorption line in previous data}

Extensive Monte-Carlo simulations (10k runs) were carried out, producing simulated spectra with the FAKEIT routine in XSPEC, to estimate the significance of the $\mathrm{C} 10$ redshifted absorption line. An absorbed power-law with the best-fit parameters obtained from the observed data was used as input model. The simulated spectra were fitted first with a powerlaw model, then a narrow absorption Gaussian line has been added to the model. The absorption line energy has been left free to vary in the range $2-7 \mathrm{keV}$ as well as the line (negative) intensity. About 30 of the simulated spectra shows a $\Delta$ Cash $>12$, the value observed in the C10 XMM-Newton spectrum between a fit without and with line. Therefore we estimate that the probability to detect the observed $\Delta$ Cash by chance in the XMM-Newton data alone is $\sim 3 \times 10^{-3}$, i.e. the feature is significant at confidence level $\sim 99.7 \%(3 \sigma)$. The significance is strengthened by the detection of the line also in ChandraACIS at $2.2 \sigma$ (Figure2).

\subsection{Statistical significance of the absorption feature}

To assess whether the apparent lack of the absorption line is due to the data quality rather than a real disappearance of the line itself, extensive Monte Carlo simulations have been carried out using the FAKEIT routine within XSPEC. Two different sets of spectra were simulated: one using the clean exposure time of case $B$ and the second using the less conservative case A. The simulated spectra have the same continuum flux and background level as observed in 2011 plus an absorption iron line with the same properties of the one observed in $\mathrm{C} 10$ (i.e., same equivalent width, width and observed energy of 4.5 $\mathrm{keV})$. Moreover, even though the equivalent width measured in $\mathrm{C} 10$ for the line was constant within error bars (Figure 8 of C10), further simulations decreasing the intensity of the line from $95 \%$ to $5 \%$ (in step of $5 \%$ ) of the $\mathrm{C} 10$ value have been simulated.

The fake spectra were first fitted with a power-law model plus an absorption line with a centroid energy fixed at the XMM-Newton $\mathrm{C} 10$ value and normalization free to vary to both positive and negative values.

The intensity of the line has been measured in all the simulated spectra, as well as the $90 \%$ confidence value. In Figure 7. the fraction of spectra where the measured intensity of the line is either consistent with zero or lower than the $90 \%$ upper limit measured in the observed data have been computed. These values (solid and open symbols respectively) are reported as function of the input line intensity for case A (red) and $\mathrm{B}$ (blue) in both $\mathrm{eV}$ units (bottom $\mathrm{X}$-axis) and also with respect to the value in $\mathrm{C} 10$ (top $\mathrm{X}$-axis).

The analysis of the simulations allows to confirm that the case A and B spectra return statistically consistent results. For line intensity lower that $70 \%$ of $\mathrm{C} 10$, the most stringent upper limit can be obtained with case A spectra (larger number of counts) regardless of higher background contribution.

The probability that the line in the observed data has and intensity comparable with the one observed in C10 is lower than $2.3 \%$ with a more stringent limit of $1.2 \%$ obtained measuring the fraction of sources with $90 \%$ confidence value lower than the one measured in these data. The probability that the line has an intensity (at $90 \%$ confidence) comparable to $50 \%$ of the $\mathrm{C} 10$ value ranges between 5 to $15 \%$, and it becomes higher with fainter input lines reaching $20-35 \%$.

\section{DISCUSSION}

In this paper we analyzed the most recent X-ray spectrum of CID-42 obtained during mid-2011 with the XMM-Newton satellite. The goal of this long observation was to properly characterize the unprecedented redshifted, variable in energy, iron absorption line observed in previous XMM-Newton and Chandra observations.

Two different XMM-Newton spectra have been used for the analysis: one preserving almost the full length of the observation thus being affected by higher background emission but 


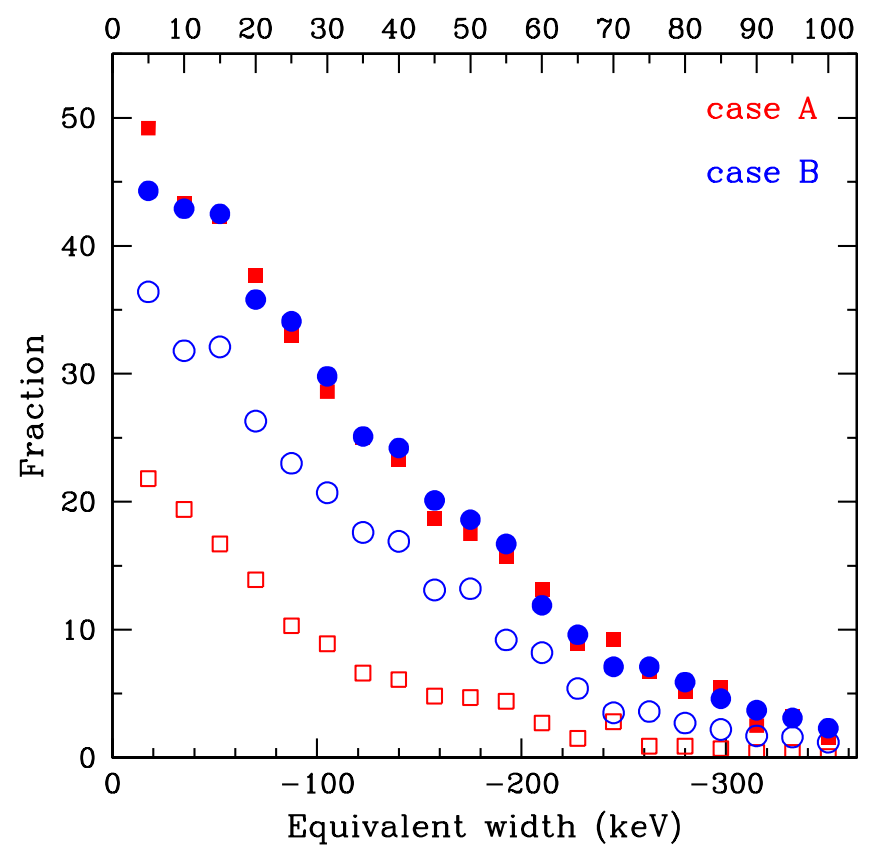

Figure 7. Fraction of simulated spectra with measured line intensity consistent with zero (solid symbols) and lower than the upper limit measured in the observed spectra (open symbols) as function of the input line intensity (lower X-axis in keV and upper X-axis in fraction of the EW in C10) for case A (red squares) and B (blue circles).

with more source counts; the second with $\sim 50 \%$ less source counts but with higher signal-to-noise ratio. The variability of CID-42, already observed in the previous 15 observations, is confirmed with these new data, showing a constant flux in the full band but the hard band flux is $20 \%$ lower than previous observations.

The previously observed inverted $P$-Cygni profile is not visible in this new observation and a limit on the equivalent width $(<162 \mathrm{eV}$ at $90 \%$ confidence level) of the absorption line is measured. The disappearance of the absorption line, detected at $3 \sigma$ in previous XMM-Newton and Chandra observations, may be explained by the combination of several factors: high background radiation, fainter continuum flux and a change in the intrinsic properties of the inflowing material.

The analysis of extensive Monte Carlo simulations return consistent results for case A and B simulated spectra. Moreover, an EW larger than the measured upper limit should have been detected with a probability of at least $98.8 \%$ if the line had the same intensity of the one measured in C10. Therefore, the higher background and faint continuum flux are not enough to explain the non-detection of the absorption line in the new spectrum, implying changes in the properties of the inflowing material.

The simulations results indicate that the line intensity could be lower than $50 \%$ of C10 intensity with a probability of $90 \%$.

CID-42 has been interpreted by $\mathrm{C} 10$ as a $\mathrm{GW}$ recoiling $\mathrm{SMBH}$ or as a system of 2 SMBHs moving away one from the other for slingshot effect. In this second scenario, C10 explained the X-ray absorption line as an outflow produced by the foreground active SMBH at the rearer one, which illuminates the otherwise undetectable wind. However, given the absence of X-ray emission from one of the two nuclei, C12 proposed that the most likely explanation for CID-42 is the one of a recoiling SMBH moving out from the galaxy at a velocity of $\sim 1300 \mathrm{~km} / \mathrm{s}$, thus the outflow scenario for the X-ray absorption line cannot be further supported. Detailed simula- tions support the recoiling scenario interpretation (Blecha et al. 2013).

In the recoiling scenario, the absorption feature is interpreted as due to absorption by ionized iron located in the proximity of the recoiling SMBH and falling with relativistic velocity into it. As the SMBH recoils from the center of the galaxy, the regions close to the $\mathrm{SMBH}$, with velocities greater than the recoil velocity, are dragged along ${ }^{\text {T2 }}$

The equivalent width of the absorption feature in $\mathrm{C} 10 \mathrm{im}-$ plied a high column density of the absorber $\left(\mathrm{N}_{H} \sim 5 \times 10^{23}\right.$ $\mathrm{cm}^{-2}$ ), and the modest absorption in the soft band required the absorber to be highly ionized $(\log \xi \sim 3)$. The disappearance of both redshifted and blueshifted absorption lines is not unusual and have been seen in many examples in the literature (Tombesi et al. 2010). To justify the disappearance of the line in this work data, assuming the same ionization parameter given that no obscuration is seen in the soft band, the column density of the absorber should have now dropped by at least a factor $10\left(\mathrm{~N}_{H}<5 \times 10^{22} \mathrm{~cm}^{-2}\right)$. Vice versa, assuming the same density of the absorber of $\mathrm{C} 10$, the ionization parameter should be $\log \xi>4$ in order to not detect the absorption line (e.g., the gas has to be extremely ionized).

A strong increase of ionization parameter can be generally associated to an increase of flux from the ionizing source, however, in CID-42 the X-ray flux is overall decreasing (see Fig. 5). A delay between the flux burst and the increase of ionization state is though expected depending on the distance between the cloud and the central source. Adopting a velocity law for the gas, as in Equation 1 of Longinotti et al. (2007), a distance of the infalling cloud from the SMBH of $\sim 100-500$ gravitational radii is estimated assuming the possible range of infall velocities (0.02-0.14c, depending on the iron ionization state), the $\mathrm{BH}$ mass and the Eddington ratio reported in $\mathrm{C} 10$.

12 For CID-42, all the material within $10^{5}$ gravitational radii from the $\mathrm{SMBH}$ will move with it. 
This distance translates into a light travel time of 4-20 $\times 10^{4} \mathrm{~s}$ (in the observer frame), which is very short and implies that the source should have experienced a strong decrease in ionizing flux in just few tens of hours before our observation, which is highly unlikely. A change in ionization state is then less preferred than a change in the density of the gas to explain the variability of the absorption line.

It is possible also to derive the life time of the infalling gas of $0.3-11 \times 10^{6} \mathrm{~s}$ (in the observer frame). This lifetime is significantly lower than the time elapsed between the X-ray observations, but larger than the duration of each observation. If the infalling material is clumpy and made of discrete clouds, in each observation a different cloud is then observed, thus its properties do not need to be related with the properties of the cloud/absorption line in previous observations. If the infalling gas is instead a continuous flow, peaks with different density must occur to produce absorption lines with changing properties.

Tombesi et al. (2010) proposed that absorption lines observed at energies below the FeK neutral line could also be interpreted as blueshifted transitions of helium or hydrogen like ions from elements lighter than iron $(\mathrm{Si}, \mathrm{S}, \mathrm{Ar}, \mathrm{Ca})$, with very high outflowing velocities $(0.1-0.5 c)$. In this scenario, given that CID-42 absorption line was observed at $\mathrm{E}_{\text {rest }}=5.86$ $\mathrm{keV}$, the outflowing velocity of the absorber would be 0.3 $0.7 c$, which is very unlikely.

A redshifted absorption line with variable properties between different observations has been observed also in source E1821+643 (Yaqoob \& Serlemitsos 2005, Jiménez-Bailón et al. 2007), candidate recoiling SMBH using optical polarized spectra (Robinson et al. 2010). The redshifted absorption line in E1821+643 has been observed in both Chandra and XMMNewton spectra as for CID-42. If a connection between the presence of variable $\mathrm{X}$-ray absorption lines and a recoiling SMBH exists, it could be the subject of further investigation and modeling.

\section{SUMMARY}

The results on the analysis of the XMM-Newton observations of the absorption line previously observed in CID-42 returns only a limit of $\mathrm{EW}<162 \mathrm{eV}$. The probability that the line intensity is not the same of $\mathrm{C} 10$ is $98.2 \%$, with a probability of $90 \%$ that the line has now an intensity of $50 \%$ of $\mathrm{C} 10$ or less. Therefore the non-detection of the line cannot be explained by high background or lower continuum but requires variability of the absorber properties, most likely variability of the absorber density, which is expected given the nature of these transient features.

Probing the gas kinematics and dynamics in the region closest to the SMBH is fundamental to understand the geometry and the accretion mode of the SMBH, in particular in the interesting case of CID-42 where the SMBH is believed to move away from the center of the galaxy. New observations are indeed needed to understand the nature of this absorption line: a monitoring of the source, with multiple long ( $100 \mathrm{ks})$ observations would be ideal to follow the variability of the absorbing material and of the broad-band continuum flux.

Absorption (blue and red shifted) features in X-ray data are an interesting subject for AGN studies as these are signature of high velocity material in the proximity of the SMBH, possibly responsible for mechanical feedback between SMBH and galaxy. However these features are intrinsically variable thus highly debated and difficult to observe at the limit of the capability of CCD and gratings (only for few local sources) instru- ments. Future missions as Athena+ could open a new window on this subject allowing to perform more detailed modeling and collect statistical samples of these features (Georgakakis et al. 2013).

The authors thanks A. Longinotti for useful discussions and the anonymous referee for the useful suggestions to improve the overall interpretation of this source. F.C., S.M. and G.L. acknowledge support by the NASA contract 11-ADAP110218. K.J. acknowledges support by the German Science Foundation (DFG), grant Ja 1114/3-1. S.K. research was supported by the DFG cluster of excellence "Origin and Structure of the Universe" (www.universe-cluster.de). M.E. and S.K. thank the Aspen Center for Physics for support and hospitality. The Aspen Center for Physics is supported by the National Science Foundation under Grant No. PHYS-1066293. A.C. acknowledges financial contribution from the agreement ASI-INAF I/009/10/0 and INAF-PRIN 2011

\section{REFERENCES}

Arnaud, K. A. 1999, Bulletin of the American Astronomical Society, 31, 734 Batcheldor, D., Robinson, A., Axon, D. J., Perlman, E. S., \& Merritt, D. 2010, ApJ, 717, L6

Bekenstein, J. D. 1973, ApJ, 183, 657

Blecha, L., Civano, F., Elvis, M., \& Loeb, A. 2013, MNRAS, 428, 1341

Blecha, L., Cox, T. J., Loeb, A., \& Hernquist, L. 2011, MNRAS, 412, 2154

Bonning, E. W., Shields, G. A., \& Salviander, S. 2007, ApJ, 666, L13

Brusa, M., Civano, F., Comastri, A., et al. 2010, ApJ, 716, 348

Cappelluti, N., Brusa, M., Hasinger, G., et al. 2009, A\&A, 497, 635

Cappelluti, N., Hasinger, G., Brusa, M., et al. 2007, ApJS, 172, 341

Cappi, M., Tombesi, F., Bianchi, S., et al. 2009, A\&A, 504, 401

Cash, W. 1979, ApJ, 228, 939

Civano, F., Elvis, M., Brusa, M., et al. 2012, ApJS, 201, 30

Civano, F., Elvis, M., Lanzuisi, G., et al. 2012, ApJ, 752, 49

Civano, F., Elvis, M., Lanzuisi, G., et al. 2010, ApJ, 717, 209

Colpi, M., \& Dotti, M. 2009, arXiv:0906.4339

Dadina, M., Cappi, M., Malaguti, G., Ponti, G., \& de Rosa, A. 2005, A\&A, 442, 461

Elvis, M. 2000, ApJ, 545, 63

Elvis, M., Civano, F., Vignali, C., et al. 2009, ApJS, 184, 158

Eracleous, M., Boroson, T. A., Halpern, J. P., \& Liu, J. 2012, ApJS, 201, 23

Fiore, F., Puccetti, S., Grazian, A., et al. 2012, A\&A, 537, A16

Georgakakis, A., Carrera, F., Lanzuisi, G., et al. 2013, arXiv:1306.2328

Grupe, D., Komossa, S., Gallo, L. C., et al. 2012, ApJS, 199, 28

Guedes, J., Madau, P., Mayer, L., \& Callegari, S. 2011, ApJ, 729, 125

Hopkins, P. F., Hernquist, L., Cox, T. J., \& Kereš, D. 2008, ApJS, 175, 356

Jiménez-Bailón, E., Santos-Lleó, M., Piconcelli, E., et al. 2007, A\&A, 461, 917

Jonker, P. G., Torres, M. A. P., Fabian, A. C., et al. 2010, MNRAS, 407, 645 Kalberla, P. M. W., Burton, W. B., Hartmann, D., et al. 2005, A\&A, 440, 775 King, A. R., \& Pounds, K. A. 2003, MNRAS, 345, 657

Komossa, S. 2012, Advances in Astronomy, 2012,

Komossa, S., Zhou, H., \& Lu, H. 2008, ApJ, 678, L81

Lanzuisi, G., Civano, F., Elvis, M., et al. 2013, MNRAS, 431, 978

Loeb, A. 2007, Physical Review Letters, 99, 041103

Longinotti, A. L., Sim, S. A., Nandra, K., \& Cappi, M. 2007, MNRAS, 374, 237

Matt, G., Porquet, D., Bianchi, S., et al. 2005, A\&A, 435, 857

Merritt, D., Storchi-Bergmann, T., Robinson, A., et al. 2006, MNRAS, 367, 1746

Nandra, K., George, I. M., Mushotzky, R. F., Turner, T. J., \& Yaqoob, T. 1999, ApJ, 523, L17

Nandra, K., O’Neill, P. M., George, I. M., \& Reeves, J. N. 2007, MNRAS, 382, 194

O’Neill, P. M., Nandra, K., Cappi, M., Longinotti, A. L., \& Sim, S. A. 2007, MNRAS, 381, L94

Peres, A. 1962, Physical Review, 128, 2471

Ponti, G., Cappi, M., Costantini, E., et al. 2013, A\&A, 549, A72

Pounds, K. A., \& Reeves, J. N. 2009, MNRAS, 397, 249

Reeves, J. N., Pounds, K., Uttley, P., et al. 2005, ApJ, 633, L81

Robinson, A., Young, S., Axon, D. J., Kharb, P., \& Smith, J. E. 2010, ApJ, 717, L122

Shields, G. A., Bonning, E. W., \& Salviander, S. 2009, ApJ, 696, 1367

Sijacki, D., Springel, V., \& Haehnelt, M. G. 2011, MNRAS, 414, 3656

Steinhardt, C. L., Schramm, M., Silverman, J. D., et al. 2012, ApJ, 759, 24

Tombesi, F., Cappi, M., Reeves, J. N., et al. 2013, MNRAS, 430, 1102

Tombesi, F., Cappi, M., Reeves, J. N., et al. 2010, A\&A, 521, A57

Vaughan, S., \& Uttley, P. 2008, MNRAS, 390, 421 
Lanzuisi et al.

Volonteri, M., Haardt, F., \& Madau, P. 2003, ApJ, 582, 559

Yaqoob, T., \& Serlemitsos, P. 2005, ApJ, 623, 112 